Cahiers de recherches médiévales

\title{
Les poisons de Jacques Cœur
}

De la mort d'Agnès Sorel aux méfaits de la fraude

\section{Pierre Prétou}

\section{(2) OpenEdition \\ Journals}

Édition électronique

URL : https://journals.openedition.org/crm/11509

DOI : $10.4000 / \mathrm{crm} .11509$

ISSN : 1955-2424

Éditeur

Honoré Champion

\section{Édition imprimée}

Date de publication : 15 juin 2009

Pagination : 121-140

ISSN : $1272-9752$

\section{Référence électronique}

Pierre Prétou, «Les poisons de Jacques Cœur », Cahiers de recherches médiévales [En ligne], 17 | 2009, mis en ligne le 15 juin 2012, consulté le 15 décembre 2022. URL : http://journals.openedition.org/crm/ 11509 ; DOI : https://doi.org/10.4000/crm.11509 


\title{
RM
}

\section{Les poisons de Jacques Cour : De la mort d'Âgnès Sorel aux méfaits de la fraude}

\begin{abstract}
In 1451, at the hour of the victorious triumph of King Charles VII, a suspicion of poisoning following the death of Agnès Sorel ruined the splendour of the court. Initially accused of the murder, Jacques Coeur, the King's argentier, fell two years later, but for other crimes connected with lese-majesty. This astonishing case calls for a reintrpretation that links poison with fraud, and the poisoning of the King's household with the reform of the kingdom. At Jacques Coeur's trial, it was really the fair and unfair uses of gold that were being judged, and in a climate of pernicious rumour. The edict of Lusignan, even though it acquitted him on the charge of murdering of Agnès Sorel, found Jacques Coeur guilty of another poisoning : that of fraudulent gold created by his activities and that royal rhetoric fashioned after the seizure. The assimilation of gold and poison justified a reordering of the kingdom, inspired by the notion of venom and favourable to the strengthening of French royal power.
\end{abstract}

Résumé: En 1451, à l'heure du triomphe victorieux du roi Charles VII, une suspicion de venin consécutive au trépas d'Agnès Sorel anéantit le lustre de la cour. Initialement accusé du fait, l'Argentier Jacques Cour tombe deux ans plus tard, mais pour d'autres forfaits réunis par les mots du crime politique. L'étonnante affaire nécessite une relecture qui relie le poison à la fraude et l'intoxication de la maison du roi à la réformation du royaume. Au procès Jacques Cour, ce sont les usages justes et injustes de l'argent qui avaient été jugés, propagés par la rumeur vénéneuse. Lors de l'arrêt de Lusignan, bien qu'ajourné sur le venin, Jacques Cour n'était pas innocent d'un autre poison, celui des ors frauduleux générés par ses activités et que la rhétorique royale fit surgir d'une mise en sujétion comptable. Or et poison ainsi assimilés, justifièrent une remise en ordre du royaume, inspirée par le venin et propice à l'élévation de la Majesté française.

En ce temps le Roy estoit fort enamourez d'une gente et belle damoiselle, que on nommoit lors communement par le Royalme la Belle Agnes, et depuis le Roy lui fist donner le nom de damoiselle de Beaute. Et il lui fut dit que Jaques Cuer avoit empoisonné ou fait empoisonner ladicte demoiselle; et duquel empoisonnement elle alla de vie a trespas. ${ }^{1}$

Mathieu d'Escouchy, Chronique, Chap. CXXX.

Dans l'encre du venin réputé avoir emporté la Dame de Beauté, nombre de plumes firent d'une mort toxique, un joyau d'histoire de France enchâssé dans le cercle de l'infortune qui accabla l'Argentier du roi, jusqu'à sa condamnation en 1453. L'association de deux faits, l'anéantissement de l'emblème de la beauté aimée du roi d'une part, le procès de la personnification de l'argent d'autre part, fit de l'affaire Jacques Cœur un objet d'histoire remarquable dans lequel le poison se

${ }^{1}$ G. Du Fresne de Beaucourt, Chronique de Mathieu d'Escouchy, tome II, Paris, Société d'Histoire de France, 1863, p. 283-284.

Cahiers de Recherches Médiévales, 17, 2009 
mêlait au motif de la Roue de Fortune, au triomphe de Charles VII, à la construction de l'État et à l'essor du négoce ${ }^{2}$. Toutefois, l'écriture historique, prompte à s'emparer de Jacques Cœur afin de faire émerger les contradictions opposant condition marchande et service du roi, s'évertue encore à dissocier les deux événements, poison charnel et fraude d'argent, réduisant ainsi la part de la mort toxique à celle d'une simple attaque ad hominem qui avait opportunément abattu le serviteur du roi.

Crime de poison, crime énorme, crime de plagiat ou de prison privée, crime de fraude, crime de faux-monnayage, crime d'intelligence avec les Sarrasins : l'arrêt de condamnation de Lusignan de 1453 additionne les chefs d'accusation sans pour autant les réunir explicitement dans une seule et même qualification. Cette abondante liste de méfaits contenue dans le procès Jacques Cœur fut ensuite recueillie dans les collections érudites de l'époque moderne, collections qui recensaient alors les anciens procès impliquant le crimen maiestatis ${ }^{3}$. Toutefois, si la lèse-majesté est bien citée dans l'acte, c'est le crime de venin qui englobe l'affaire : il ouvre, puis ferme la rédaction de la sentence, après avoir circulé dans toutes les informations criminelles. À suivre la logique linéaire de l'acte, le poison déclenchait et ordonnait toute l'affaire. Cet écho venimeux semble être confirmé par les nombreuses relations des chroniqueurs du $\mathrm{XV}^{\mathrm{e}}$ siècle, relations qui associèrent dans une même affaire les deux perditions d'Agnès Sorel et de Jacques Cœur ${ }^{4}$. Au portrait de l'empoisonneur retenu par les chroniques, les biographies récentes de l'Argentier préférèrent toutefois celui de l'homme d'affaires, une figure de l'ascension sociale fondée sur le pouvoir de l'argent au milieu du $\mathrm{XV}^{\mathrm{e}}$ siècle français ${ }^{5}$. C'est occulter que l'étude des actes du procès révèle des particularités peu intelligibles ${ }^{6}$. Jacques

\footnotetext{
${ }^{2}$ Propos sensible dans P. Clément, Jacques Cour et Charles VII ou la France au XV siècle, Paris, Librairie académique, 1863. Jacques Cœur y devint un emblème de l'esprit d'entreprise opposant les thuriféraires de l'État autoritaire et les partisans du capitalisme libéral bourgeois. ${ }^{3}$ J. Chiffoleau, «Le crime de majesté, la politique et l'extraordinaire. Note sur les collections érudites de procès de lèse-majesté du XVII ${ }^{\mathrm{e}}$ siècle français et sur leurs exemples médiévaux », Y. M. Bercé (dir.), Les procès politiques (XIV $-X V I I^{e}$ siècle), Rome, École française de Rome, 2007, p. 577-662.

${ }^{4}$ Nous avons utilisé les éditions suivantes: Mathieu d'Escouchy, Chronique, éd. G. Du Fresne de Beaucourt, Paris, Société d'Histoire de France, 3 tomes, 1863; Thomas Basin, Histoire de Charles VII, éd. Ch. Samaran, Paris, Les Belles Lettres, 2 tomes, 1965 ; J. du Clercq, Mémoires, éd. J.A. Buchon, Paris, 1826 ; Jean Chartier, Histoire du roy Charles VII, éd. Valet de Viriville, tome II, Paris, 1858, Jean Juvénal des Ursins, Ecrits politiques, éd. P. S. Lewis, Paris, 1978, 1985, 1992, 3 vol.

${ }^{5}$ Michel Mollat insista sur la condition marchande du personnage, tandis que Jacques Heers privilégia le serviteur du roi. Kathryn Reyerson, plus mesurée, s'est efforcée de faire se rejoindre ces deux dimensions. Signalons donc M. Mollat, Jacques Cour ou l'esprit d'entreprise, Paris, Aubier Montaigne, 1988 ; sa contradiction dans J. Heers, Jacques Cour, Paris, Perrin, 1997 ; ainsi que la synthèse de K. Reyerson, Jacques Cœur, Entrepreneur and King's Bursar, New York, Pearson Longman, 2005.

${ }^{6}$ Le procès de Jacques Cœur a fait l'objet d'une étude méticuleuse : $\mathrm{R}$ Guillot, Le procès de Jacques Cour (1451-1457), Bourges, Caisse nationale des Monuments historiques et des Sites, 1974. Signalons également la mise au point récente de K. Reyerson, «Le procès de
} 
Cœur fut certes accusé d'empoisonnement, mais la sentence ne l'en rendit pas coupable. Comment alors interpréter l'abondante liste de crimes que la condamnation survenue à Lusignan en 1453 associe à l'occasion d'une rhétorique judiciaire fondée sur le crime de poison? Le Journal du Procureur Dauvet, célébré par les historiens de l'économie pour la rigueur de son inventaire témoigne bien d'un effort d'enquête ainsi que d'une lecture judiciaire et morale des biens du condamné. Quel sens donner à ces écritures comptables réunies sous le terme, sans doute impropre, de «Journal»? Cet effort d'appropriation et de délimitation des droits du roi faisant suite à la sentence ${ }^{7}$, l'enquête semble avoir eu lieu après la condamnation. Force est donc de constater que si l'affaire Jacques Cœur paraît bien connue, les logiques de la démonstration exemplaire produite par la justice royale ouvrent ici une possible relecture du cas qui relie la rumeur de poison à l'enrichissement injuste ${ }^{8}$.

Innocent du poison qui emporta Agnès Sorel, Jacques Cœur l'était-il de la fraude introduite par son or dans le royaume? Le venin, point de départ de l'accusation, ne s'efface pas aussi rapidement que l'on veut bien le penser. Il s'infiltre dans les informations criminelles, s'agrège avec le faux monétaire avant de se coaguler dans la fraude d'argent. Jacques Cœur fut rendu coupable de nombreux crimes envers son souverain, coupable d'avoir corrompu la domesticité du roi, et à travers elle son royaume. Cela, le procureur Dauvet se devait de le démontrer, en distinguant, partout où il enquêta, la part altérée des biens de Jacques Cœur qui devait revenir au souverain. À travers l'assimilation de l'or au venin introduit dans les veines commerciales du pays, c'est la place de l'argent qui était jugée au procès. Du poison à l'honneur et aux biens du roi, il est un chemin que les chroniques, les actes de justice et le Journal du Procureur Dauvet nous invitent à parcourir. Partie du venin, la justice royale avait établi une analogie entre or et poison. Cette analogie avait permis aux accusateurs de rencontrer le crime énorme, avant de consolider son existence de manière comptable : après l'avoir qualifié, la justice royale matérialisa le forfait dans la fraude des biens du roi. Si la preuve du venin ayant corrompu le corps d'Agnès Sorel n'avait pu être rapportée, celle de la corruption du royaume fut démontrée. Jacques Cœur en était l'évident coupable.

\section{Le venin de l'innocence coupable}

Chroniques comme actes de procédure font de la rumeur vénéneuse le point de départ de l'affaire Jacques Cœur. Agnès Sorel, favorite du roi, avait été emportée dans la journée du 11 février 1450. Jeune, belle, choyée par le souverain, la disparition de la Dame de Beauté surprit les contemporains. Une mort violente, improbable, et finalement injuste, qui devait attirer sur elle le soupçon du venin. Le corps sans vie d'Agnès Sorel devint un objet d'interrogations politiques portées par

Jacques Cœur», Y. M. Bercé (dir.), Les procès politiques (XIVe-XVII siècle), Rome, École française de Rome, 2007, p. 123-144.

${ }^{7}$ M. Mollat, Les affaires de Jacques Cour, Journal du Procureur Dauvet, Paris, Armand Colin, 2 tomes, 1953.

${ }^{8}$ En empruntant ici les pistes ouvertes par: F. Collard, Pouvoir et poison, histoire d'un crime politique de l'Antiquité à nos jours, Paris, Seuil, 2007. 
un regard courtisan ${ }^{9}$. Cui bono ? Mis à part le Dauphin et à défaut de cabale clairement identifiable, la rumeur porta le nom de l'Argentier du roi comme ayant, de près ou de loin, eut un lien coupable avec ce trépas. Celui qui faisait commerce d'apparat auprès de la Cour fut ostensiblement désigné comme responsable de la destruction du joyau domestique de la couronne. Fragilisé par la haine de ses adversaires, l'Argentier ne se sentait pourtant pas menacé, pensant qu'il «estoit aussi bien envers le roy que il avoit jamais este, quelque chose que on en $\operatorname{dist}^{10} »$. Proche de la Dame de Beauté, celle-ci avait désigné Jacques Cœur pour être son exécuteur testamentaire. Etait-il de son parti ? $^{11} \mathrm{Si}$ un lien existait entre Agnès et l'officier, il n'était pas pour autant une accusation en bonne et due forme, jusqu'à ce qu'une dame de la Cour, Jeanne de Vendôme, vienne porter haut le crime en l'imputant à l'Argentier. À suivre Mathieu d'Escouchy, l'affaire devint clameur entraînant le déchaînement de la colère royale et de la procédure inquisitoire.

La réaction du roi fut immédiate et l'on procéda d'office : Jacques Cœur fut arrêté, pris de corps et de biens, mis en prison à Taillebourg, en attente d'informations criminelles qui feraient la lumière sur le cas. Puisqu'elle était survenue dans la sphère de la familiarité du roi, le souverain conserva l'affaire auprès de lui, déployant ici la puissance de sa justice retenue. Les chroniqueurs insistent sur le fait que l'accusation portée par Jeanne de Vendôme avait «enflammé » le cœur du roi en raison de l'amour qu'il portait à sa dame. Toutefois, les informations diligentées ne permirent pas d'établir la culpabilité de Jacques Cœur. La rumeur, toujours réversible, n'accablait pas unanimement l'homme. Ainsi que l'écrit Thomas Basin en incise: licet revera de hoc a pluribus crederetur immunis $^{12}$. Pire, les experts convoqués auprès du corps sans vie déclarèrent que la présumée victime était en réalité morte d'un «flux de ventre ${ }^{13}$. Le médecin du roi lui-même, Robert Poitevin, écarta fermement la probabilité du venin puisqu'Agnès, enceinte, avait donné la vie à un enfant qui, sain de tout poison, lui avait survécu. La persistance de la suspicion auprès de la paléopathologie ne laisse pas d'étonner. L'utilisation récente des méthodes de la médecine légale - ou pathographie - sur les restes présumés d'Agnès Sorel illustre cette fascination irrationnelle qu'exerce encore sur nos contemporains ce mystère d'histoire nationale cristallisant la renommée vénéneuse. En 2005, les études pratiquées sous la direction de Philippe Charlier ont permis d'identifier les restes de la Dame de Beauté, en tous points conformes - âge, apparence et nombre de grossesses comprises - aux relations du $\mathrm{XV}^{\mathrm{e}}$ siècle $^{14}$. La cause de la mort rapportée par l'enquête est une intoxication lourde au mercure, imputée à la maladresse d'un traitement médiéval inapproprié. La médecine du XXI ${ }^{\mathrm{e}}$ siècle défiant celle du $\mathrm{XV}^{\mathrm{e}}$ siècle, c'est le médecin du roi, Robert Poitevin, qui se trouve désormais soupçonné pour avoir administré un vermifuge qui

\footnotetext{
${ }^{9}$ Les positions de Jean II Juvénal des Ursins et de Thomas Basin sur Agnès Sorel rappellent qu'il convient d'apporter quelques nuances à l'émotion courtisane.

${ }^{10}$ Cf. R. Guillot, Le procès de Jacques Cœur, op. cit., p. 27.

${ }^{11}$ Nous ne trancherons pas cette question à laquelle le procès en réhabilitation introduit sous Louis XI apporte bien des réponses.

${ }^{12}$ Thomas Basin, Histoire de Charles VII, éd. Ch. Samaran, op.cit., p. 282.

${ }^{13}$ Jean Chartier, Histoire du roy Charles VII, éd. Valet de Viriville, op. cit., tome II, p. 186.

${ }^{14}$ Cf. P. Charlier, Médecin des morts : récits de paléopathologie, Paris, Fayard, 2006.
} 
réduit l'histoire politique à l'étude technique d'une pharmacopée et, donc, à bien peu de choses. Jacques Cœur, Louis XI, Robert Poitevin, les coupables défilent selon les époques mais est-ce bien important? Quel apport pour le scandale qui fit surface au milieu du XV $\mathrm{XV}^{\mathrm{e}}$ siècle ? Excessivement faible, car ce n'est pas tant la nocuité du venin qui importe, que sa propagation politique. L'analyse paléopathologique conforte finalement l'idée simpliste selon laquelle un coup bas avait été porté par les seuls adversaires de Jacques Cœur. La grandeur de la majesté royale réduite à la matérialité d'un vermifuge coupable, voilà une démarche que nous écarterons.

Particulièrement rompu à l'exercice qui consiste à relativiser une accusation criminelle en la réduisant à une œuvre partisane, Thomas Basin nous livre sa compréhension de l'affaire: par le poison, on avait intoxiqué l'opinion du roi et de sa Cour. L'évêque de Lisieux procéda ici comme il le fit pour Jeanne d'Arc, que Charles VII lui avait demandé de réhabiliter. Des délateurs stipendiés - ou submissis delatoribus $^{15}$ - affidés aux adversaires de Cœur, s'employèrent à faire tomber l'Argentier. Les «haineulx » étaient visiblement nombreux et les sources en dressent la copieuse liste. Il s'agissait essentiellement des rivaux de Cœur, soit dans les affaires commerciales, soit dans le clientélisme politique. Portée par Jeanne de Vendôme, l'accusation était également étayée par les marchands de Montpellier et de Toulouse. Un grand nombre de débiteurs en délicatesse trouvait aussi avantage dans la manœuvre. Au premier rang de ces adversaires, émerge la figure d'Otto Castellani qui eut tout le loisir d'accabler son rival puisque le roi décida de l'investir d'une partie de l'enquête. C'est toutefois un autre marchand, Michel Teinturier, qui lui porta le coup de grâce en invoquant les Sarrasins au procès, Infidèles qui firent de Cour l'ennemi de la Chrétienté universelle. Ces gens firent grande diligence, opiniâtres et préoccupés qu'ils étaient de s'assurer de la perte de leur ennemi, quitte à encourager la justice retenue à déployer contre lui la procédure extraordinaire. Le fait confirme ici que le poison constitue une arme politique réversible, parfois utilisée pour tuer, parfois pour accuser d'avoir tué ${ }^{16}$. Le simple fait de soumettre l'adversaire aux rigueurs de la procédure extraordinaire était en soi une très belle réussite. Indéniablement, entraver toute instruction à décharge et se voir confier la conduite de la procédure l'était plus encore: Charles VII livrait son Argentier aux mains de ses adversaires. Doit-on pour autant réduire la chute de Jacques Cœur à la seule qualité des perfides manœuvres orchestrées contre lui ? L'instrumentalisation du poison ne fait aucun doute, mais elle ne suffit pas à expliquer le crime énorme et l'ampleur prise par l'affaire, sauf à considérer, comme le fait Thomas Basin, que le scandale politique ne repose communément que sur l'aléatoire destinée de ceux qui croient rencontrer la faveur royale: talem exitum sepe invenisse, qui regum ac principum familiaritates acquirere studuerunt ${ }^{17}$. Ce soupçon de pensée morale, ajouté à ses propos qualifiant la beauté adultérine d'Agnès Sorel, achève de convaincre le lecteur qu'il y avait là une forme de cycle politique : une alternance d'ascensions et de déchéances affectant les favorites et favoris. La Dame répandait des usages impudiques à la Cour, Jacques Cœur en faisait autant avec l'argent. Tout était factice, leur chute simultanée était regrettable certes, mais tellement prévisible.

${ }^{15}$ Thomas Basin, Histoire de Charles VII, éd. Ch. Samaran, op.cit., p. 284.

${ }^{16} \mathrm{~F}$. Collard, Le crime de poison au Moyen Âge, Paris, PUF, 2003, p. 233-276.

${ }^{17}$ Thomas Basin, Histoire de Charles VII, éd. Ch. Samaran, op.cit., p. 286. 
Notons que Jean Chartier conduit une analogie similaire lorsqu'il condamne tour à tour mondanités et rapines, «excessifz atours» et «convoitise ${ }^{18}$ », unissant ainsi Agnès Sorel et Jacques Cœur dans une même réprobation. Toutefois, que la Roue de Fortune tourne inlassablement pour les favoris vaniteux, ne nous dispense pas d'observer des fondements politiques solides dans le scandale ouvert en 1451.

L'accusation formulée par Jeanne de Vendôme abattit l'honorabilité de Jacques Cœur. En observant les composantes néfastes de la délation, on en mesure toute la portée et la relation de Mathieu d'Escouchy éclaire plus fortement ce venin que celle de Thomas Basin. Le chroniqueur met en relief les origines de Jacques Cour, « homme de petite generacion ${ }^{19}{ }$ - c'est-à-dire extraction - qu'il oppose à la beauté altière et à la noblesse de la victime. Thomas Basin reprend le motif - ex plebeyo genere $e^{20}$ - ainsi que Jacques du Clercq - «petite generation sans quelque noblesse $^{21} \gg$. Toutefois, seul Mathieu d'Escouchy s'engage plus avant lorsqu'il fait suivre cette origine de la rumeur selon laquelle Cœur s'enrichissait « sur le denier du $\operatorname{Roy}^{22} »$. Jacques Cœur, ainsi chargé d'une renommée de parvenu, souffrit ensuite d'une autre rumeur, bien plus dévastatrice, alimentée par ses ennemis et selon laquelle il avait empoisonné Agnès Sorel. Le feuilleté de paroles calomniatrices relevées par le chroniqueur indiquait que derrière l'admirable ascension de Jacques Cœur, se cachaient peut-être des méthodes perfides. En conséquence, la place de l'argent dans le royaume, ainsi que le pouvoir et les faveurs que la richesse permettait d'obtenir, précédaient le venin avant de s'y agréger subtilement. Le lustre que la présence d'Agnès Sorel apportait à la Cour était donc placé en contrepoint d'un autre apparat, celui qui était issu du monopole des produits de luxe de la Cour avec lequel Jacques Coeur faisait fortune. Toutefois, la figure de l'une s'opposait en tous points à la réputation de l'autre. Le procédé n'étonne pas, bien au contraire il prospère sur la contamination de la fama personae par la fama facti: le fait d'empoisonner vient clairement aggraver les traits de personnalité de l'Argentier ${ }^{23}$. Tous les chroniqueurs s'accordent pour présenter les origines de Jacques Cœur avant la rumeur de poison mortel. Le lecteur du récit sait déjà que le trouble suscité par l'ascension sociale permise par l'argent l'emportera dans l'explication de l'affaire de poison. Dès lors, il importe peu qu'Agnès Sorel ait été, ou non, empoisonnée, et Mathieu d'Escouchy se trahit lorsqu'il expose la rumeur du venin avant même que sa «damoiselle de Beaute» n'en périsse. Jacques du Clercq, précautionneux et favorable à la belle, choisit de ne pas évoquer le poison, même s'il cite Jeanne de Vendôme en omettant de rapporter la nature de l'accusation qu'elle porta. Le venin semble alors perdre de sa valeur dans le récit, certains évoquant l'innocence de Cœur. Pourtant, l'arrêt de condamnation ne permet pas d'aller aussi loin. La justice du roi n'innocenta pas l'Argentier de ce persistant venin : elle l'ajourna. «Et au

\footnotetext{
${ }^{18}$ Jean Chartier, Histoire du roy Charles VII, éd. Valet de Viriville, op. cit., p. 181, p. 328.

${ }^{19}$ Mathieu d'Escouchy, Chronique, éd. G. Du Fresne de Beaucourt, op. cit., p. 280.

${ }^{20}$ Thomas Basin, Histoire de Charles VII, éd. Ch. Samaran, op.cit., p. 150.

${ }^{21}$ Jacques du Clercq, Mémoires, éd. J. A. Buchon, op. cit., p. 84.

${ }^{22}$ Mathieu d'Escouchy, Chronique, éd. G. Du Fresne de Beaucourt, op. cit., p. 282.

${ }^{23}$ Sur la fama: T. Fenster et D. Lord Smail (éds.), Fama, The Politics of Talk and Reputation in Medieval Europe, New York, Cornell University Press, 2003 et C. Gauvard (dir.), «La Renommée », Médiévales, n² 24, 1993.
} 
regard des poisons, pour ce que le proces n'est pas en estat de juger pour le present, nous n'en faisons a present aucun jugement, et pour cause ${ }^{24} \gg$. Le texte renvoyait l'établissement du crime à de plus sérieuses preuves que celles qui avaient été obtenues. Pour autant, Jacques Cœur n'était pas blanchi de la mort d'Agnès Sorel. Il était toujours soupçonné car le poison pouvait encore être utile à la condamnation. À l'issue de la sentence interlocutoire de Chissay, le venin fut dissocié de l'instruction principale, gisant dans un autre sac que l'on ne parvenait pas à remplir ${ }^{25}$. Désormais, deux contenants récoltaient parallèlement les actes de procédure : d'un côté la fraude d'argent et de l'autre le venin. Si au final, l'arrêt de Lusignan en 1453 ne retient pas la mort d'Agnès Sorel pour fonder la culpabilité de Cœur, il n'en reste pas moins qu'elle se fige dans le texte, à la manière d'une tache indélébile. Quelle utilité pouvait bien avoir ce triste rappel ? Sur ce cas, l'Argentier bénéficiait visiblement d'une forme paradoxale d'innocence coupable, remise à plus tard, et qui conduisait l'ensemble de la charge criminelle.

D'évidence, la mort d'Agnès Sorel, ainsi que la rumeur d'empoisonnement, avaient fait offense au roi, tant et si bien qu'il fallait un coupable pour vider le cas. Le poison avait donc permis d'imputer à l'accusé toutes sortes de comportements coupables marqués par la falsification. Là était sans doute la principale vertu de la suspicion. Avait-on seulement fait preuve d'opportunisme? Non, car les manœuvres des «haineulx » n'expliquent pas l'ampleur prise par l'affaire et le venin ne se réduit pas à la seule matérialité de la substance mortelle : son introduction dans la Maison $\mathrm{du}$ roi impliquait une issue politique, un chemin qui mènerait à l'ensemble du royaume. Dans le secret de la Cour, la procédure inquisitoire en élabora alors la rhétorique fatale.

\section{Les rhétoriques toxiques}

Aurait-on voulu simplement abattre Jacques Cœur que lui retirer la faveur du roi y aurait sans doute suffit, mais, dans cette affaire se jouait un contenu bien plus large. L'Argentier fut rendu coupable d'une multitude de «grans crimes et malefices ${ }^{26} »$ initiés par le venin. Par ce procédé, les écritures judiciaires, en associant le poison à la fraude, créèrent une image implicite: l'Argentier avait empoisonné la Cour comme le royaume. Occulté par la beauté et la fortune, le criminel était falsificateur car en lieu et place des produits de luxe et de la saine monnaie, l'on devinait force dettes et sombres agissements. Cette image implicite explique que l'empilement des crimes suivait un ordonnancement démonstratif: même innocent de la mort de la Dame de Beauté, Jacques Cœur n'en était pas moins coupable d'un autre poison qui avait contaminé, à travers le corps et la maison du roi, l'ensemble du royaume. En associant l'ensemble des faits reprochés, le jugement fondait une confluence criminelle rendant nécessaire la superposition des faits, emportés ensemble dans une même logique ${ }^{27}$. Suivons ici cet ordonnancement.

${ }^{24}$ Arrêt de condamnation, éd. R. Guillot, Le procès de Jacques Cour, op. cit., p.114.

${ }^{25}$ Cf. R. Guillot, Le procès de Jacques Couur, op. cit., p. 42-44.

${ }^{26}$ Journal du Procureur Dauvet, éd. M. Mollat, op. cit., p. 18.

${ }^{27}$ Rappelons ici la recension finale de ces délits en insistant sur la grande porosité de ces qualifications : «Crimes de concussion et exaccions de noz finances et de noz païs et subgiez, de faulx, de transport de grant quantite d'argent aux Sarrazins, ennemys de la foy chrestienne 
L'arrêt de condamnation amorce la charge criminelle par la clameur consécutive à la mort d'Agnès Sorel, clameur qui fit apparaitre les crimes d'or et de commerce commis par Jacques Cour avec la complicité des Sarrasins. Une fois le crime établi, l'acte l'objective alors par l'évaluation comptable des fraudes de l'accusé. Cette logique est également celle des chroniqueurs qui, tous, s'attardent sur le fait qui matérialisa le crime énorme: la vente d'armes et la réduction d'un chrétien égyptien en esclavage. Les derniers interrogatoires procèdent à l'inverse : l'accusé fut d'abord questionné sur ses fraudes commerciales avant que celles-ci ne conduisent les officiers à l'interroger sur sa complicité avec les Sarrasins. L'information s'éleva crescendo, de la fraude au crime énorme, avant de revenir sur le détail des concussions de l'accusée ${ }^{28}$. Le point d'orgue de toute l'affaire, évoqué par tous les chroniqueurs, fut cet enfant chrétien, réduit à l'esclavage en terre d'Egypte. Pour ce cas, les rigueurs les plus abouties de la procédure extraordinaire furent employées, dans une alternance éprouvante d'intimidations, de tortures et de confrontations. Retenue dans le secret de la Cour, la procédure inquisitoire ne fut que rarement interrompue par sentence interlocutoire. Si elle advint à Chissay en 1452, permettant ainsi à Jacques Coeur de tenter de se défendre, c'était pour mieux légitimer l'usage de la procédure extraordinaire.

L'énoncé des crimes faisait du poison le point de départ de l'accusation. En le rappelant, le texte suit ici une stricte chronologie procédurale mais cet énoncé vient se heurter aussitôt au second fait reproché qui lui succède : le faux-monnayage. L'or trouvait là toute sa place, à la manière d'un écho sinistre qui rappelait habilement les origines de Jacques Cour. Le faux-monnayeur était un criminel qui avait le tort de faire apparaitre de la valeur là où elle n'aurait pas dû être. Faisant écho aux écrits de Nicolas Oresme, les justices françaises avaient pris l'habitude d'objectiver la fausse monnaie par l'évaluation du poids et de l'aloi du métal précieux, avant de les sanctuariser auprès de l'intérêt public garanti par le roi ${ }^{29}$. L'Argentier, alors qu'il avait pris à ferme la monnaie de Bourges, avait été gracié en 1429 pour avoir fait «prouffit de vint ou trente escus pour marc, ou il n'en devait avoir que deux ecus, en desfraudant nous et la chose publicque de nostre royaume et en commectant en ce faisant crime de faulse monnoye ${ }^{30} »$. L'élévation de Jacques Cour se trouvait ainsi dépréciée par les stigmates de la tromperie aggravée endommageant le bien public. Pipeur en fait d'or, puisque son ascension en provenait, toute son honorabilité était atteinte. Pourtant, tout comme le poison, ce deuxième crime ne pouvait être reproché à Jacques Cœur car il avait obtenu des lettres de rémission pour ce fait. Le crime était remis certes, mais pas aboli. Comme ce souvenir demeurait, il établissait encore la suspicion de culpabilité. L'association

et de nous, de transport de billon d'or et d'argent en grant nombre hors de nostre royaume, transgression des ordonnances royaulx, crime de lese-maieste et autres crimes. » Journal du Procureur Dauvet, éd. M. Mollat, op. cit., p. 13.

${ }^{28}$ La justice royale était, à cette date, particulièrement expérimentée en matière de fraude. En témoigne l'affaire Jean de Saincoins : Jean Chartier, Histoire du roy Charles VII, éd. Valet de Viriville, op. cit., p. 244.

${ }^{29}$ Nicolas Oresme, Traité des monnaies, éd. C. Dupuy, Paris, La Manufacture, 1989.

${ }^{30}$ Arrêt de condamnation de Jacques Cour, ADL, Duché de Roannais, E² ; édition R. Guillot, Le procès de Jacques Cour, op. cit., p. 109. 
du poison et du faux-monnayage est ici très nette. Celui qui connaît les arts minéraux et les secrets de la mort par substances, connaît également les poudres qui permettent la contrefaçon. Notons que Jacques Cœur avait lui-même procédé à des enquêtes en fait «d'arquemie ${ }^{31} »$. Poison, alchimie ${ }^{32}$, faux-monnayage venaient ainsi converger afin de blâmer une expertise aurifère néfaste, toute consacrée à la tromperie lâche. Il s'agissait ici d'un comportement inverse de la noblesse attendue par la Cour, noblesse que des lettres de Charles VII avaient pourtant autrefois conférée à son Argentier pour valider son élévation sociale. Mais l'Argentier avait obtenu des lettres de rémission qu'il produisit. Pourquoi alors avoir encore retenu ce crime pardonné dans l'arrêt de condamnation ? Parce qu'il venait opportunément alourdir le cas en suggérant la récidive du fait ${ }^{33}$. Nécessairement, l'énoncé criminel se devait d'accrocher sur quelque crime aurifère que l'on pût cette fois imputer au coupable sans déjuger les pardons antérieurs. Les évasions de métal précieux en Orient vinrent à propos et le commerce méditerranéen de Jacques Cœur ouvrit le troisième chef d'accusation. En jouant sur les cours de l'argent et de l'or au Levant, l'officier était parvenu à trouver une solide source de profit. Hélas, cette source était orientale, notoirement néfaste, et aggravait par conséquent la réputation d'empoisonneur et de falsificateur de l'Argentier. Les poisons sont réputés venir de l'est ${ }^{34}$. L'or également. Et les Sarrasins d'entrer fictivement au prétoire.

Jacques Cœur, en commerçant avec l'Orient méditerranéen, se rendit coupable d'évasion frauduleuse de métaux précieux. Les interrogatoires révélèrent l'âpreté avec laquelle il récoltait toutes sortes de billons afin de couler les lingots d'argent utiles au commerce levantin ${ }^{35}$. Ce faisant, il avait permis que le précieux métal du royaume se perde dans les bourses sarrasines alors que le royaume en avait un besoin pressant ${ }^{36}$. Pire, il avait fait apposer une fleur de lys sur le produit de sa forge, exposant ainsi l'honneur du roi aux mains des étrangers. Or, les témoins insistèrent pour rapporter que ces lingots étaient tous de mauvais titre: Cœur trompait les marchands sous couleur d'une frappe royale. Toutefois, le fait fut contesté par le coupable, lui qui estimait donner «bruit au navigaige des

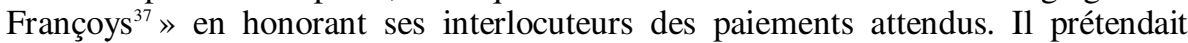
avoir voulu conférer du lustre au commerce des galées de France, pour les profits et

\footnotetext{
${ }^{31}$ M. Mollat, Jacques Coeur ou l'esprit d'entreprise, op. cit., p. 389.

${ }^{32}$ Sur ce point, nous renvoyons à C. de Mérindol, «Jacques Cœur et l'alchimie», Le crisi dell' alchimia, Micrologus. Natura, scienze e societa medievali, 3, 1995, p. 263-278.

${ }^{33} \mathrm{Si}$ la récidive de la désobéissance implique sans doute la trahison, ce mot est toutefois absent de l'arrêt de condamnation. Nous ne pouvons donc rejoindre sur ce point l'interprétation du procès en matière de trahison: S. H. Cuttler, The Law of Treason and Treason Trials in Later Medieval France, Cambridge, Cambridge University Press, 2003, p. 209.

${ }^{34}$ Nous rejoignons ici les conclusions de F. Collard, «Une arme venue d'ailleurs. Portrait de l'étranger en empoisonneur », L'étranger au Moyen Âge, $\mathrm{XXX}^{\mathrm{e}}$ Congrès de la SHMES, Publications de la Sorbonne, 2000, p. 95-106.

${ }^{35}$ Journal du Procureur Dauvet, édition M. Mollat, op. cit., p. 7.

${ }^{36}$ Sur les famines monétaires du Moyen Âge tardif, nous renvoyons à P. Spufford, Money and its use in Medieval Europe, Cambridge, 1988, p. 339 et s.

${ }^{37}$ Interrogatoires. R. Guillot, Le procès de Jacques Cæeur, op. cit., p. 88.
} 
honorabilités du roi. Néanmoins, l'accusation pencha en faveur des détracteurs de Cœur qui rapportaient que les Sarrasins «avoient dit tout communement et en presence de plusieurs autres marchands estranges que François estoient trompeurs $^{38} \gg$. Les activités monétaires de Cœur contaminaient donc l'honneur de la personne même du roi ${ }^{39}$. Le raisonnement était extensible au royaume puisque, dans le discours monétaire, la circulation de la monnaie était comparée au bon écoulement du sang d'un pays. Ainsi aux États généraux de Tours en 1484, le commun tirait grief du fait que «ce royaume à présent est comme un corps qui a été évacué de son sang par diverses saignées, et tellement que tous ses membres sont vidés. Et comme ainsi soit que le sang est le soutènement de la vie corporelle, aussi sont les finances du royaume le soutènement de la chose publique ${ }^{40} \gg$. La monnaie était ici assimilée au sang d'un corps dont le commerce constituait les veines. La fausse monnaie était donc un venin. De même que l'argent et la santé corporelle s'associaient étroitement, de même le faux monnayage et le poison s'acoquinaient dangereusement. Or, cette fois, l'or pipé de Jacques Cour avait bel et bien corrompu la santé monétaire du pays à l'occasion d'une infraction qui n'avait pas encore été jugée et qui faisait écho à la rémission obtenue en 1429 pour crime de faussemonnaie. Certes la marque contrefaite «sans congie ni licence» ne possédait pas la gravité du faux monétaire complet, mais l'accusation se révélait d'une extrême utilité car cette fois, elle permettait de relier définitivement poison et faux monnayage. Dès lors, une fenêtre s'entrouvrait qui n'attendait que d'être agrandie: la complicité avec les Sarrasins. En deux mots : le crime énorme, qualificatif évident que l'acte n'allait pas tarder à poser explicitement.

Une fois énoncé le commerce des lingots contrefaits, l'accusation s'attarda sur le rôle des Infidèles dans les activités de Cour. En toute logique, les écritures quittent alors le domaine aurifère pour observer le rôle des ennemis de la Chrétienté et ainsi faire de l'Argentier leur complice puisqu'il «avoit renye Dieu ${ }^{41} »$. Le commerce de Cœur, déjà accablé, favorisait d'évidence les adversaires du Christ. La preuve en fut amenée dès lors qu'il fut démontré que les galées de l'Argentier embarquaient des marchandises que les Sarrasins utilisaient pour occire les Chrétiens. Les lingots d'argent contrefaits cédaient ici la place au trafic illicite d'armes. Le raisonnement était étroit, car dépendant d'un fragile récit circonstancié. L'on disait que Jacques Cœur, afin de plaire au Sultan d'Egypte, avait offert à ce dernier quelques harnois de bonne facture. L'accusé confessa ce cadeau commercial, dont il minimisa l'importance et la portée. Toutefois, il avait bien armé les ennemis de la foi et pour parer sa défense, l'on rapporta que l'on avait entendu dire que le Sultan avait remporté une bataille sur les Croisés grâce à ces armes. L'affirmation

\footnotetext{
${ }^{38}$ Arrêt de Lusignan. Journal du Procureur Dauvet, éd. M. Mollat, op. cit., p. 8.

${ }^{39}$ Sur l'honneur du roi et l'articulation des peines, cf. C. Gauvard, «L'honneur du roi. Peines et rituels judiciaires au Parlement de Paris à la fin du Moyen Âge », C. Gauvard, R. Jacob (dir.), Les rites de la justice au Moyen Âge, Paris, Le Léopard d'or, 2000, p. 99-123.

${ }^{40}$ États généraux de Tours, 1484. Cahier présenté au roi et son conseil des trois États touchant le bien, utilité et profit du royaume et de la chose publique. Chapitre du commun ; cité dans G. Depeyrot, Histoire de la monnaie des origines au XVIII siècle, t. 2, Wetteren, Moneta, 1995, p. 278.

${ }^{41}$ Arrêt de condamnation. R. Guillot, Le procès de Jacques Couur, op. cit., p.110
} 
paraîtra fantasque, mais elle était bien trop utile au procès pour ne pas surgir des informations. Peu à peu, le crime énorme se caractérisait mais il fallait appuyer cette rhétorique sur un fait net et bien renseigné, fait qui aurait établi que les activités de Jacques Cœur entraînaient la perdition des Chrétiens. L'affaire de l'esclave réfugié sur les galées offrit cette opportunité. Rappelons-en ici les principaux éléments. À Alexandrie, un facteur de Jacques Cœur avait donné asile à un esclave, un enfant qui se disait chrétien. Réfugié sur les galées de France, l'enfant fut ramené au royaume mais Cœur, craignant que ses interlocuteurs alexandrins n'en prennent ombrage, le fit renvoyer de force auprès de ses maîtres égyptiens. L'accusation tenait enfin son incontestable crime énorme. Et les mots du crime politique de s'y engouffrer ${ }^{42}$ : «crime, grans et enormes crimes, comme de leze majeste, force publicque, prison privee, transport de nostre juridiction en autre, crime de playe et autres ${ }^{43} \gg$. Jacques Cœur, en livrant un enfant chrétien à la vindicte infidèle, avait fauté envers le Christ. Brusquement, le ton de l'arrêt change car, d'évidence, l'autorité licite avait été gravement heurtée. L'on enquêta alors sur les motivations de Cœur en dégageant deux causes qui ne manquèrent pas de caractériser la tyrannie. En effet, on fit la preuve que l'Argentier n'était préoccupé que par son profit, celui de son commerce avec les Egyptiens. Son avarice était si grande qu'il n'aurait pas souffert que l'on payât un prix modique de 50 ducats, au regard du crime ici commis, pour affranchir l'enfant. Pire, Jacques Cœur était entré en grande colère en apprenant la présence de l'enfant. Il injuria ses facteurs, «leur fit mauvaise chiere», «renia Dieu», et les menaça de voie de fait, jetant ici toute son autorité pour faire aboutir sa décision déraisonnable. Si son facteur Teinturier ne pliait pas, «il le destruiroit». Ainsi, les mots de la fureur firent-ils que l'enfant fut ramené et livré, achevant le portrait en tyrannie que le crime énorme supposait. La procédure extraordinaire se déploya contre le coupable, et se fit particulièrement insistante sur l'affaire de l'enfant égyptien. On «en sauroit la verite par sa bouche par voye extraordinaire de question $^{44} \gg$. Jacques Cœur, en invoquant le privilège de clergie, avait bien tenté de se soustraire aux extrémités de la torture mais il avait été débouté. On présenta donc l'accusé devant billots et sellettes. Apeuré, il déclara que «s'il est gisne il confessera ce que on vouldra ${ }^{45} »$. Malheureusement, cette déclaration confirma l'intérêt qu'il y avait à employer la torture contre lui afin d'obtenir l'aveu ${ }^{46}$. Lié, très fortement éprouvé, l'Argentier céda et s'en remit aux témoins à charge, ses «haygneux », validant ainsi l'ensemble des mots de la fureur.

$\mathrm{Si}$ les derniers interrogatoires avaient eu raison de la résistance de l'Argentier, il parvenait encore à nier le poison. Il ne vit pas qu'insidieusement, l'instruction le menait droit vers une toute autre qualification. L'information criminelle, en investissant le champ lexical de la tyrannie, s'ouvrait ici sur une forêt

\footnotetext{
${ }^{42}$ Sur les actes nefandi: J. Chiffoleau, «Dire l'indicible: remarques sur la catégorie du nefandum du XI $\mathrm{I}^{\mathrm{e}}$ au XV $\mathrm{XV}^{\mathrm{e}}$ siècle », AESC, 1990, p. 289-324.

${ }^{43}$ Arrêt de Lusignan. R. Guillot, Le procès de Jacques Cæur, op. cit., p. 110.

${ }^{44}$ Arrêt de Lusignan. R. Guillot, Le procès de Jacques Cour, op. cit., p. 113.

${ }^{45}$ Interrogatoires. R. Guillot, Le procès de Jacques Cour, op. cit., p. 93.

${ }^{46}$ Sur l'aveu, cf.: J. Chiffoleau, «Avouer l'inavouable: l'émergence de la procédure inquisitoriale au Moyen Âge », R. Dulong (dir.), L'aveu, histoire, sociologie, philosophie, Paris, PUF, 2001, p. 57-98.
} 
de termes caractérisant le pouvoir injuste, d'exercice comme d'usurpation, si bien que l'imposture fit son entrée ${ }^{47}$. Logiquement, l'arrêt de condamnation aborde alors les abus d'autorité de Cœur. La démonstration était impeccable: l'Argentier avait coutume de s'emparer de la légitime puissance de son souverain. En Languedoc, il commit de grands excès de «force publicque». Il y abusait de la Presse par laquelle il recrutait de force les marins qui lui étaient nécessaires en détournant un ordre royal qui lui faisait mandement de poursuivre «les coquins et les ruffiens $»^{48}$. Cette tyrannie d'exercice, pleine de fureur, avait eu des conséquences abominables : ici des sergents du roi avaient été embarqués par erreur, là un pèlerin allemand avait été réduit à la condition de galérien - chose horrible - car, " de dueil et desplaisance s'estoit gecté en la mer et noyé ${ }^{49} \gg$. Détournement d'autorité, confusion dans la foi, Jacques Cœur avait retourné les faveurs du roi contre son souverain. Il tirait même profit de celle-ci en vendant et monnayant son influence auprès du roi à ceux qui espéraient de son entregent qu'il fit se conclure de bons mariages. Jacques Cœur était «meut de grant avarice ${ }^{50} »$. On rapporta qu'il la justifiait en privé en commettant crime de parole contre son roi. Et les témoins de rapporter que l'accusé badinait en affirmant que l'argent requis l'était pour le plaisir du jeu de dés du roi, «pour jouer au dez», «pour son jeu des festes ${ }^{51} »$. Il ne restait plus qu'à fonder la tyrannie d'usurpation afin de faire surgir la probabilité d'un complot. L'arrêt de condamnation énonce alors le crime qui coiffait cette patiente élaboration : Jacques Cœur avait contrefait le sceau même du roi. Des perquisitions avaient en effet révélé l'existence de pouvoirs falsifiés, des signatures royales imitées, ainsi qu'une imitation contrefaite du petit sceau royal. Les preuves de l'usage de ces faux manquèrent et Jacques Cœur répliqua en insistant sur le fait qu'il n'avait pas employé ces procédés, que les contrefaçons se fondaient en réalité sur des missions confiées par ordre du roi. L'Argentier sentit ici le grand péril dans lequel il était, aussi récusa-t-il avoir falsifié l'autorité de Charles VII, torture ou pas, renvoyant la responsabilité des signes de validation falsifiés à ses gens qui «le faisoient sinon par esbatement et pour passer temps ${ }^{52} »$ sans mal y penser. Il n'y avait rien de sérieux làdedans, mais l'on n'arrête pas aisément la progression d'une accusation en crime énorme offensant la majesté. L'autorité entière de Cœur était contrefaçon et pour mieux l'appuyer, l'on rappelait à l'envi que ses gens agissaient sans fidélité ni aveu honorable ${ }^{53}$. «La pluspart ne le avoient oncques veu » ajoute Jacques du Clercq ${ }^{54}$. À

\footnotetext{
${ }^{47}$ Sur les contours de l'imposture: G. Lecuppre, L'imposture politique au Moyen Âge: la seconde vie des rois, Paris, PUF, 2005, p. 23-58.

${ }^{48}$ Interrogatoires. R. Guillot, Le procès de Jacques Cour, op. cit., p. 95.

${ }^{49}$ Arrêt de condamnation. R. Guillot, Le procès de Jacques Cour, op. cit., p. 110-111.

${ }^{50}$ Arrêt de condamnation, R. Guillot, Le procès de Jacques Coeur, op. cit., p. 111.

${ }^{51}$ Interrogatoires. R. Guillot, Le procès de Jacques Couur, op. cit., p. 105. Sur le crime de parole : C. Leveleux, La parole interdite : le blasphème dans la France médiévale, Paris, De Boccard, 2001.

${ }^{52}$ Interrogatoires. R. Guillot, Le procès de Jacques Couur, op. cit., p. 99.

${ }^{53}$ Un propos à relier aux mots qui qualifient l'absence de fidélité des tueurs à gages: C. Gauvard, «La violence commanditée : la criminalisation des «tueurs à gages » aux derniers siècles du Moyen Âge », Annales, $62^{\mathrm{e}}$ année, $\mathrm{n}^{\circ}$ 5, 2007, p. 1005-1029.

${ }^{54}$ Jacques du Clercq, Mémoires, éd. J. A. Buchon, op. cit., p. 84.
} 
trop vouloir nier avoir fait usage privé des signes de l'autorité royale, l'Argentier ne put s'opposer aux mots du pouvoir injuste et de la fureur. Certes, il réfutait l'intention coupable, déclarant par exemple ne pas avoir su que l'enfant égyptien était chrétien, mais il se mit insuffisamment en défense contre la personnalité coupable qui émergeait lentement des instructions ${ }^{55}$.

L'accusation, amorcée par la rumeur du venin, empruntait donc un chemin étroit ponctué de bien maigres preuves. Toutefois, ici ou là, de petits récits édifiants alimentaient le crime politique. Le trait était certes forcé mais la rhétorique judiciaire déployée par la justice retenue était implacable. Du poison, le crime avait glissé vers le faux-monnayage; du faux-monnayage, on passait aux profits illicites du commerce extérieur; de ce dernier, on basculait en direction de la complicité avec les Infidèles, complicité qui validait un crime énorme étayé par un portrait en tyrannie, d'exercice comme d'usurpation. Les officiers avaient donc enquêté sur Jacques Cœur comme s'il eut tous les traits de l'empoisonneur: tromperies, enormia, falsifications, pouvoir de méfaire sous l'apparence du bien. Ce faisant, ils avaient relié le venin à l'argent, tout en ouvrant une analogie implicite entre la santé monétaire d'un royaume et celle d'un corps. Au final, ils restauraient le royaume en dévoilant les crimes cachés de l'Argentier. Restait à refermer l'ensemble de la procédure en évaluant l'intensité du méfait. Une appropriation comptable fit alors place à la charge criminelle : la fraude devait être chiffrée afin d'être certifiée et concrétisée. L'arrêt de condamnation, après avoir imputé la contrefaçon du sceau, retint alors les fraudes simples du profit du roi. Si la portée pénale de ces dernières était moins grande que les crimes énormes évoqués, elles n'en venaient pas moins matérialiser et chiffrer le crime. La fraude d'argent concluait donc l'accusation sur une ouverture comptable appropriative de la majesté offensée.

\section{«Desfrauder » le royaume}

Jacques Cœur n'était qu'un sombre falsificateur que l'enquête se devait de mettre en lumière. La rhétorique judiciaire déployée par l'arrêt de condamnation avait privilégié un crescendo dont le point d'orgue avait été le sort de l'enfant égyptien. Toutefois, le crime politique venait ensuite se perdre dans la longue liste des fraudes consécutives au droit de marque ou à la collecte de la Gabelle du Languedoc ${ }^{56}$. Afin de mieux appuyer le cheminement qui, partant de la clameur vénéneuse, s'était achevé sur le crime politique, les chroniqueurs ne retinrent pas ces délits. Néanmoins, du point de vue de la justice royale, l'évaluation de la fraude se révélait décisive. Cet énoncé comptable permettait une appropriation du crime énorme, désormais chiffré, pour ne pas dire déchiffré. L'Argentier était condamné, mais il n'était pas utile de le mettre à mort ni de faire usage de l'éclat des supplices. Tout l'intérêt de la condamnation reposait dans l'écriture comptable de la fraude, écriture qui permettait de restaurer la juste majesté en rétablissant l'autorité du roi sur les parties corrompues du royaume. Cette mission là, qui consistait à consolider les conclusions de l'information criminelle, fut confiée au procureur Jean Dauvet.

\footnotetext{
${ }^{55}$ Interrogatoires. R. Guillot, Le procès de Jacques Cæur, op. cit., p. 91-98

${ }^{56}$ Nous ne nous étendrons pas sur le détail de celles-ci en renvoyant aux excellentes analyses de M. Mollat, Jacques Cour ou l'esprit d'entreprise, op.cit., p. 219-316 et de R. Guillot, Le procès de Jacques Cœur, op. cit., p. 57-82.
} 
Le 29 mai 1453, Jacques Cœur fut principalement condamné à amende honorable, bannissement, et à deux versements, l'un de 100.000 écus pour ses concussions, et l'autre de 300.000 écus pour le profit du roi. Simultanément, l'Argentier et son accusatrice, Jeanne de Vendôme, eurent à demander pardon à Dieu, au roi et à la justice car les arrêts, en statuant sur le cas des deux personnes impliquées par les instructions, concernaient bien les poisons au premier chef. La grandeur de la neutralité royale s'honorait du lustre de la miséricorde consentie au souverain pontife qui la réclamait pour son protégé Cœur: l'objectif poursuivi n'impliquait pas nécessairement la peine de mort. Toutefois, l'ampleur de la somme visée supposait que le condamné soit retenu en prison pour dettes, jusqu'à paiement libératoire ${ }^{57}$. Là commence la mission du procureur Dauvet: exécuter un arrêt de condamnation $^{58}$. Investi de pouvoirs extraordinaires par Charles VII, l'officier devait en réalité enquêter sur les biens de Jacques Cœur afin de les réserver au roi et d'ainsi garantir le paiement de la prodigieuse amende infligée au condamné. Paradoxalement, les affaires de Jacques Cœur, affaires qui avaient tant retenu les informations criminelles, ne seraient donc clairement identifiées qu'une fois la sentence exécutée. De 1453 à 1457 , le procureur veilla à la créance du roi en arpentant le royaume à la recherche des facteurs et affaires de l'Argentier. La manière avec laquelle il le fit répandit grandement la nouvelle de la condamnation de l'Argentier. Autant l'instruction s'était déroulée dans le secret de la Cour, autant l'exécution du jugement fut rendue publique par l'artifice des innombrables criées créancières $^{59}$. Puisqu'il fallait liquider les avoirs du coupable, Jean Dauvet faisait «crier et publier a son de trompe ${ }^{60} »$ en chacune des places où il se rendait, une fois vérifiés les usages locaux en matière de droit des biens. «Oyez de par le Roy nostre sire $^{61} \gg$ entendait-on, puis l'arrêt de condamnation était rappelé. L'on criait deux fois, parfois trois pour obtenir que les sujets déclarassent les biens de Cœur en leur possession. Lorsque des enchères publiques se révélaient utiles, elles étaient également criées, toujours dans la forme évoquée. L'exécution de l'arrêt, à défaut de supplices exemplaires, mobilisa ainsi l'opinion publique avec autant de force qu'un échafaud $^{62}$. Simultanément, cette notoriété des mainmises et séquestres permettait d'isoler les affaires de Cœur, comme l'on met de côté des parties gâtées du royaume.

\footnotetext{
${ }^{57}$ Sur la prison pour dettes, et les usages de la justice royale en la matière : J. Claustre, «La dette, la haine et la force : les débuts de la prison pour dette à la fin du Moyen Âge », Revue historique, CCCIX/4, 2008, p. 797-821, ainsi qu'à l'édition de sa thèse : J. Claustre, Dans les geôles du roi: L'emprisonnement pour dette à Paris à la fin du Moyen Âge, Paris, Publications de la Sorbonne, 2007.

${ }^{58}$ Lettres de commission du procureur Dauvet. Journal du Procureur Dauvet, éd. M. Mollat, op. cit., p. 15-21.

${ }^{59}$ Sur le cri, cf. D. Lett et N. Offenstadt (dir.), Haro ! Noël! Oyé ! : pratiques du cri au Moyen Âge, Paris, Publications de la Sorbonne, 2004.

${ }^{60}$ Journal du Procureur Dauvet, éd. M. Mollat, op. cit., p. 125.

${ }^{61}$ Ibid., p. 126.

${ }^{62}$ Une autre méthode d'ouverture d'un espace public confirmant les propos de C. Gauvard et $\mathrm{P}$. Hamon, «Les sujets du roi de France face aux procès politiques $\left(\mathrm{XIV}^{\mathrm{e}}-\mathrm{XVI}^{\mathrm{e}}\right.$ siècle) », Y. M. Bercé (dir.), Les procès politiques (XIV -XVII siècle), Rome, École française de Rome, 2007, p. 479-511.
} 
Partout, le cri de la préemption du roi brisait le secret des affaires de l'Argentier tout en faisant connaître sa condamnation ${ }^{63}$. Pour autant, le procureur Dauvet ne tolérait aucune expression dissidente. Investi de pouvoirs exceptionnels, il faisait arrêter, emprisonner, il menait les interrogatoires en bonne forme, assortissait l'authenticité des déclarations de lourdes amendes et même condamnait. «Les droiz et auctorite du Roy sont si notoires de non pouvoir enfraindre sa main ne exploicter sur icelle sans son congie et licence» indique le procureur au détour de son journal ${ }^{64}$. Beaucoup de débiteurs s'enfuirent alors et, mois après mois, mis en échec par un grand nombre de défauts, la pratique de Jean Dauvet se durcit avec succès lorsqu'il invoque le châtiment afflictif sur les désobéissants, la "peine d'estre pugniz comme de fur et larrecin $»^{65}$. Partis du corps d'Agnès Sorel corrompu par le poison, les officiers du roi avaient donc trouvé le chemin qui menait au chevet du corps souffrant de l'État. En isolant les affaires de Jacques Cœur, on faisait œuvre de rétablissement de l'autorité royale. Par la même occasion, la notoriété des contours de l'État fut éclaircie. Le journal du procureur prit même les détours d'une enquête en souveraineté, lorsque, instruisant dans les régions frontalières, il délimita les limites du royaume de France, face au roi de Sicile, à la Bretagne, au Bourbonnais, et en démêlant les avoirs des «subgects et justiciables» de Charles VII avec les villes de Naples et de Gênes. En Provence, il rencontra le roi René qui lui refusa le droit d'instrumenter, récusant ainsi toute sujétion à son voisin. Nec erat patria subalienata avec France ${ }^{66}$. Dauvet dut se contenter de la réponse et tolérer que les coutumes de Marseille interrompent la conduite de sa charge. Toutefois, il l'emporta dans bien des places des marches de France, fit plier nombre de hauts personnages, parmi lesquels le Duc de Bourbon, dont il fit arrêter le trésorier, avant d'enjoindre à sa Chambre des Comptes de Bourbonnais de ne plus empiéter sur les droits du roi ${ }^{67}$. Ainsi l'honneur du roi était-il clairement délimité, tant à l'intérieur qu'à l'extérieur. Cette délimitation résultait des écritures comptables du procureur Dauvet, écritures propices à la mise en sujétion des pays qu'il arpentait.

Le procureur zélé fut en tout soutenu par le roi, obtenant de lui les chartes et les entrevues qu'il souhaitait ${ }^{68}$. Charles VII était satisfait car Jean Dauvet dévoilait les sinuosités occultes du réseau de Jacques Cœur. Créance de l'un, transport de marchandises de l'autre, aveu d'un voiturier: l'officier suivait chaque trace. La difficulté de la mission s'accentuait à mesure que l'on découvrait que les gens de Cœur répugnaient à établir les dettes en bonne forme, préférant la parole confiante, quand bien même les créanciers rappelaient-ils que «on ne sait qui meurt ne qui vit $»^{69}$. Les associations de Jacques Cœur ne reposaient pas sur l'écrit mais sur les relations de gens ayant «amitie congnoissance ensemble» qui déclenchaient

\footnotetext{
${ }^{63}$ Sur les alternances entre mise au secret et publicité : J. Chiffoleau, «Ecclesia de occultis non iudicat. L'Église, le secret et l'occulte du XII ${ }^{\mathrm{e}}$ au XV $\mathrm{XV}^{\mathrm{e}}$ siècle », Il segreto nel Medioevo, Micrologus, Nature, Sciences and Medieval Societies, XIII, 2005.

${ }^{64}$ Journal du Procureur Dauvet, éd. M. Mollat, op. cit., p. 428.

${ }^{65}$ Ibid., p. 410.

${ }^{66}$ Ibid., p. 203.

${ }^{67}$ Ibid., p. 449.

${ }^{68}$ Ibid., p. 455.

${ }^{69}$ Ibid., p. 508.
} 
«parolles de compaignie $»^{70}$. À l'inverse, Jean Dauvet démêlait le tout par l'écriture comptable, mais les profits du roi se révélaient médiocres. Les principaux commis de l'Argentier - Guillaume de Varye, Jean de Village, Antoine Noir - demeuraient insaisissables malgré leur évidente «subgeccion». Insistant, le procureur enquêta fort autour des mines métallifères de l'Argentier, pensant sans doute retrouver là une des sources cachées de la fortune de Jacques Cœur. Là encore, le bilan financier fut court. Toutefois, le succès politique semblait grand, car à chaque fois que la main du roi était restaurée, ce dernier l'encourageait dans sa tâche. Ici réside sans doute la cause de la satisfaction royale. En effet, l'instrumentation de Jean Dauvet ne s'arrêtait pas aux simples recouvrements: patiemment, méticuleusement, ses écritures comptables séparaient le bien du mal, avant que d'administrer de saines prescriptions qui avaient valeur d'une réformation des usages. Jean Dauvet exigeait systématiquement l'établissement d'une comptabilité double que le monde du négoce ne s'empressait pas d'accomplir. Il rejetait les comptabilités «qui n'estoient en forme ne en ordre $»^{7_{1}}$, précisant qu'il fallait «bon langaige et y declairer les dabtes tant des recettes et despences que des ventes et amplectes ${ }^{72} \gg$. Ainsi contraints à $«$ affiner $»^{73}$, les marchands se voyaient opposer des principes rigoureux définis par «equite et bonne foy», en «bon et loyal inventaire $»^{74}$. En somme, il s'agissait bien d'une réformation royale $\mathrm{du}$ 《stille de marchans $»^{75}$. Le principe fut également appliqué aux mines du Lyonnais pour lesquelles le procureur rédigea des statuts complets dans l'espoir que cette nouvelle administration permît d'en tirer la plus «grant quantite que on pourroit ${ }^{76}$ en mettant fin à l'incurie. De même, l'on fit grand cas de l'expertise savante en matière d'or et d'argent. Une fois l'orfèvrerie de Cour confisquée et évaluée précisément par gens experts, le bruit des enchères faisait résonner une comptabilité appropriative des usages de l'argent, puisqu'elle changeait un mode d'accès négocié à la connaissance de l'or. Le prix était juste ou injuste et Dauvet retirait l'objet de la vente en cas d'écart léonin entre l'enchérissement et la somme expertisée. Après une telle remise en ordre, il ne restait qu'à récompenser les gens de négoce qui, comme Jean Billy, mettaient bon ordre et bonne diligence à obéir au procureur. Celui-ci fut absous pour ce qu'il « este renomme bon et loyal marchant $\gg^{77}$. De même, Jean Dauvet fit preuve de miséricorde envers le débiteur qui avait fait «bancque ropte et est pouvre ${ }^{78}$. Jean Dauvet distinguait les bons des mauvais, ainsi que les usages de la justice royale et le regard politique du roi le lui commandaient.

Les historiens de l'économie exploitèrent largement les registres du Journal $d u$ Procureur Dauvet, tous unanimes pour y trouver une source remarquable

${ }^{70}$ Ibid., p. 39.

${ }^{71}$ Ibid., p. 116, p. 137.

${ }^{72}$ Ibid., p. 143, p. 254.

${ }^{73}$ Ibid., p. 164.

${ }^{74}$ Lettres relatives à l'exécution de l'arrêt de condamnation de Jacques Cœur. Journal du

Procureur Dauvet, éd. M. Mollat, op. cit., p. 18.

${ }^{75}$ Journal du Procureur Dauvet, éd. M. Mollat, op. cit., p. 215.

${ }^{76}$ Ibid., p. 314 , p. 347.

${ }^{77}$ Ibid., p. 531.

${ }^{78}$ Ibid., p. 340. 
d'histoire commerciale. L'officier fut loué pour sa précision, son opiniâtreté méticuleuse et son respect des formes du droit. Peu de venin dans les mots employés par le procureur, pourtant, à bien y regarder, il semble que l'on ait exagéré la neutralité du rédacteur afin de mieux assurer les qualités du document envers l'analyse économique. D'évidence, la source était punitive, consécutive à l'action pénale et instrumentalisée par le regard politique. L'accusé, s'il n'était pas coupable de la mort d'Agnès Sorel n'en avait pas moins les traits de l'empoisonneur et du falsificateur qui méfait dans l'ombre. En mettant en lumière ces agissements, Jean Dauvet intégrait pleinement le raisonnement judiciaire et les objectifs que Charles VII avait fixés. Son enquête devait rétablir publiquement l'honneur du roi, tant dans la lettre du droit, que dans son esprit. L'officier usa donc alternativement de rigueur et de miséricorde ${ }^{79}$, conformément aux usages de France, délimitant ainsi en creux le profil du bon sujet du roi ramené à bonne obédience ${ }^{80}$. La lettre de mission de Jean Dauvet n'y faisait pas référence, mais la pratique le fit. À plusieurs reprises, le procureur accentue ou relâche la pression qui pèse sur les débiteurs, en fonction de leur attitude envers le pouvoir royal. Certes, Jean Dauvet n'avait pas le pouvoir de grâce, mais l'étroite correspondance qu'il entretenait avec le souverain lui permettait d'obtenir les pardons et les rigueurs qu'il suggérait au roi. Il n'est donc pas surprenant de voir apparaître les formules de la miséricorde dans les lignes rédigées par l'officier royal. À certains, il accordait répit et délais gracieux tandis que d'autres étaient immédiatement saisis et emprisonnés pour dette. Jean Dauvet retenait les suppliques, les charges de femme et d'enfants, la pauvreté et la nécessité de survivre. «J' ay este informe qu'il estoit pouvre ${ }^{81}$ écrivait l'officier. Tel est Jean Bourdin, réfugié en franchise, qui était «bien renomme et a grant charge de femme et d'enfans $\gg^{82}$. On ne pouvait procéder contre lui avec rigueur. Jean Dauvet retenait également la peine et la difficulté de paiement quand les débiteurs présentaient suppliques: «suppliant et requerant de luy donner delai de faire finance ${ }^{83}$, ou encore «au nom de dieu et de sa benoite passion que je aye pitie de luy et de son pouvre menage $»^{84}$. À la bonne renommée, le grand âge ${ }^{85}$, la maladie ${ }^{86}$, la nécessité ${ }^{87}$, la charge de famille, et la pauvreté s'ajoutait également le service du roi comme ce débiteur qui était «fort en debte a l'occasion de ce qu'il a este prisonnier aux Angloiz par trois foiz pour le service du roi $»^{88}$. D'évidence, l'officier appliquait ici

\footnotetext{
${ }^{79}$ C. Gauvard, "Grâce et exécution capitale : les deux visages de la justice royale française à la fin du Moyen Âge », Bibliothèque de l'École des Chartes, 153, 1995, p. 275-290.

${ }^{80}$ Le sujet idéal défini par l'usage de la grâce pour lequel nous renvoyons à C. Gauvard, «De grace especial ». Crime, État et société en France à la fin du Moyen Âge, Paris, Publications de la Sorbonne, 1991, p. 849-889.

${ }^{81}$ Journal du Procureur Dauvet, éd. M. Mollat, op. cit., p. 229.

${ }^{82}$ Ibid., p. 487, p. 491.

${ }^{83}$ Ibid., p. 103.

${ }^{84}$ Ibid., p. 178.

${ }^{85}$ Ibid., p. 252.

${ }^{86}$ Ibid., p. 155.

${ }^{87}$ Ibid., p. 275, p. 254.

${ }^{88}$ Ibid., p. 409, p. 636.
} 
les usages de la grâce royale ${ }^{89}$. L'affaire s'engageait encore mieux lorsque l'on avait été victime des mauvaises pratiques des facteurs de l'Argentier : «Ya grant pitié en son fait » écrivait alors le procureur ${ }^{90}$. Encore plus exemplaire fut la miséricorde accordée à l'un des membres de la famille Cour. Ravant Coeur avait supplié le procureur «en plorant et gemissant», invoquant sa grande pauvreté tout en ajoutant qu'il désirait désormais être un bon sujet du roi et ne plus s'opposer aux criées de son procureur ${ }^{91}$. Pour cette raison, il avait été chassé par ses frères. Il n'en fallait pas plus pour déclencher la miséricorde de l'officier prompt à ainsi relever une dissension au sein des défenseurs opiniâtres de l'Argentier. «Je luy dis et respondi qu'il ne se desconfortast point et que je ne faisoye doubte que le Roy n'eust pitie de luy ». Bien entendu, Charles VII confirma et Jean Dauvet lui fit livrer 30 écus et quelques vêtements ${ }^{92}$. A toutes fins utiles, le roi avait accordé 500 francs au procureur afin de subvenir aux besoins des Coeur et l'officier en faisait usage en distinguant les bons des mauvais. L'objectif était clair: en faisant preuve de «doulceur et d'equite ${ }^{93}$, Jean Dauvet instrumentait en contrepoint de Jacques Cœur. Par un jeu de miroir, le procureur faisait l'inverse de ce qui avait condamné l'Argentier. Transparence, dévouement, rigueur, miséricorde et bonnes formes concourraient à alourdir la charge criminelle pesant sur le condamné pour des défauts inverses. Ce n'est pas un hasard si, au détour de quelques interrogatoires, le procureur note $«$ la main trop forte $»^{94}$ de Cœur, sa «grant auctorite et puissance $»^{95}$ ou encore les pressions stipendiées ${ }^{96}$. Alors qu'il n'était qu'exécuteur, Dauvet poursuivait bien les lignes principales de l'arrêt de condamnation, aggravant ainsi le portrait de la tyrannie. Si les témoins ne rédigeaient pas un registre conforme, c'était parce que Jacques Cœur ne le «vouloit souffrir». Pour «le doubte de luy desplaire », il avait été obéi mais une fois condamné, les écritures comptables saines avaient bien entendu repris ${ }^{97}$. Pouvoir injuste donc. Déduisons que le Journal du Procureur Dauvet était bien une pièce à charge de plus à verser à l'instruction criminelle pourtant bouclée quatre ans plus tôt. Certes, l'arrêt avait été exécuté, mais cette exécution avait été assortie et accompagnée des justes remèdes de la réformation royale. D'une accusation en poison, résultait une mise en sujétion comptable du royaume mobilisé par le cri public et discipliné par les usages justes et injustes de l'argent.

$\mathrm{Au}$ royaume empoisonné par les fraudes d'argent de Jacques Cœur, Jean Dauvet avait appliqué une thériaque comptable. Rigueur et miséricorde s'appliquèrent plus aux pays qu'à la personne du condamné : évadé, Jacques Cour

${ }^{89}$ Cf. C. Gauvard, «De grace especial », op. cit., p. 895-930.

${ }^{90}$ Journal du Procureur Dauvet, éd. M. Mollat, op. cit., p. 150.

${ }^{91}$ Ibid., p. 129.

${ }^{92}$ Ibid., p. 139-140.

${ }^{93}$ Termes de l'abolition accordée à Jean Bourdin, Journal du Procureur Dauvet, éd. M. Mollat, op. cit., p. 564-570.

${ }^{94}$ Journal du Procureur Dauvet, éd. M. Mollat, op. cit., p. 434.

${ }^{95}$ Ibid., p. 540.

${ }^{96}$ Ibid.., p. 150.

${ }^{97}$ Ibid., p. 243. 
parvint à s'enfuir «exilie du ventre de son honneur $»^{98}$. La démonstration accomplie par la justice retenue ne lui avait laissé aucune chance: chaque crime imputé s'imbriquait dans un autre, plus grave, qui lui succédait tout en faisant émerger le crimen maiestatis, à l'occasion d'une addition de faits, tous mus par une même rhétorique venimeuse. L'ensemble avait été ensuite consolidé et matérialisé auprès des écritures comptables de la fraude. La liste des infractions ne prend sens que lorsque l'on suit la logique du point de départ : le crime de poison. La justice royale n'avait pas écrit au hasard et l'ordre linéaire de l'acte mérite l'attention. Du médecin, on était passé au procureur Dauvet, de la domesticité du roi, l'affaire s'était infléchie en direction de ses sujets, de l'empoisonnement de la dame de Beauté, la rhétorique avait versé dans l'examen méthodique des ors frauduleux de Jacques Cœur. Le venin circulait partout et liait les crimes retenus, ainsi devenus solidaires. Le travail des officiers était donc bien une œuvre de décontamination. Peu à peu, les mots du poison s'étaient certes effacés mais le raisonnement enclenché par le venin avait lourdement persisté. Nul doute que l'infraction poursuivie, partie de la Maison du Roi, avait finalement gagné l'ensemble du royaume. Les officiers n'avaient plus qu'à purger ce dernier des séquelles de la guerre de Cent ans en accentuant par là-même le triomphe de leur souverain. La santé de la Maison du roi était alors assimilable à celle du royaume, indissociable de ce dernier. De la beauté royale à la puissance de l'argent, un raisonnement issu du venin avait donc circulé, or et argent étant interprétés comme le sang du pays qu'il fallait soigner par la bonne administration du droit du roi. Le texte de la condamnation finale livrait les principales clefs de cette élaboration. Ouverte par le poison, la rédaction de l'arrêt s'achevait sur l'ajournement de ce dernier. Ainsi le venin encerclait-il l'ensemble de la procédure. Et les chroniqueurs de reprendre le motif, l'affaire Jacques Cœur venant en incise de la conquête de la Guyenne, incise amorcée par le poison et close par son jugement. En 1453, le crime politique se trouvait donc contenu entre les lignes de la rumeur vénéneuse, celles qui accusent, comme celles qui déchargent. Exit Jacques Cœur. Elevé par l'État, ce dernier avait fini par le dévorer, sacrifié sur l'autel de la restauration économique pour laquelle il avait tant œuvrée ${ }^{99}$. La circulation de l'argent s'en trouvait clarifiée, entre usages justes et injustes de la monnaie, nés dans la domesticité royale et nettement assimilés à la bonne circulation du sang du royaume. Incidemment, les exécutions du procureur Dauvet avaient signifié aux sujets une nouvelle façon de déchiffrer le pouvoir, tout en livrant une lecture claire des modes d'accès au pouvoir, lorsque l'on est du monde du négoce. Thomas Basin qui insistait sur la Roue de Fortune n'avait pas tort lorsqu'il insistait sur la réversibilité de l'amour du roi. Néanmoins, la place juste de l'argent était bien partie de la familiarité du souverain, et c'est le poison qui l'avait transportée jusqu'au royaume, ainsi mis habilement en sujétion par l'extensibilité vénéneuse de la matière criminelle. Célébrée par la majesté, l'affaire entame ensuite sa sortie politique pour entrer dans le conte populaire, conte qui

\footnotetext{
${ }^{98}$ Georges Chastellain, Temple de Jean Boccace, de la ruyne d'aucuns nobles malheureux par Georges son imitateur, éd. P. Clément, Jacques Cour et Charles VII ou la France au XV siècle, op. cit., tome I, p. XXI.

${ }^{99}$ Service du roi qui lui fit dire qu'il «cuidoit avoir autre salaire et triomphe» que les supplices de la torture judiciaire. Cf. R. Guillot, Le procès de Jacques Cœur, op. cit., p. 93.
} 
retint essentiellement le seul motif de la Roue de Fortune, en faisant de Jacques Cœur un emblème de Midas ingénu et déchu ${ }^{100}$. A reprendre les mots des chroniqueurs, le venin avait donc provoqué le «flux de ventre mortel», avant de provoquer «l'exil du ventre de l'honneur». Avec Franck Collard, constatons donc qu'il n'y a pas de fin aux histoires de poison.

Pierre Prétou

Université de La Rochelle

pierre.pretou@univ-lr.

${ }^{100}$ Recensions de P. Clément, Jacques Cour et Charles VII ou la France au XV siècle, op. cit., tome I, p. I-LX. 